\title{
From transmission error measurements to angular sampling in rotating machines with discrete geometry
}

\author{
Didier Remond* and Jarir Mahfoudh \\ Laboratoire de Dynamique des Machines et des Structures, UMR CNRS 5006, Institut National des Sciences \\ Appliquées de Lyon, Bâtiment Jean d'Alembert, 18, rue des Sciences, 69621 VILLEURBANNE Cedex, France
}

Received 4 November 2003

Revised 23 June 2004

\begin{abstract}
The benefits of angular sampling when measuring various signals in rotating machines are presented and discussed herein. The results are extracted from studies on transmission error measurements with optical encoders in the field of power transmissions and can be broadened to include phase difference measurements, such as torsional vibrations, and applied to control, monitoring and measurement in rotating machines with discrete geometry. The main conclusions are primarily that the use of angular sampling enables the exact location of harmonics and, consequently, the obtaining of spectral amplitude components with precision. This is always true even if the resolution of encoders is not directly related to the studied discrete geometry. It then becomes possible to compare these harmonics under different operating conditions, especially when speed varies, without changing any parameters in spectral analysis (window length, spectral resolution, etc.). Moreover, classical techniques of improving signal to noise ratio by averaging become fully efficient in the detection of defective elements. This study has been made possible thanks to the technique of transmission error measurement with optical encoders that allows the comparison of sampling procedures, based on the same raw data.

The intensive use of such transducers and the development of an original transmission error measurement technique lead to advocate the use of angular sampling in experimental measurements in rotating machines with discrete geometry.
\end{abstract}

\section{Introduction}

Nowadays, behavior characterization, monitoring and control of rotating machines require precise and reliable estimation of frequency components that are insensitive to operating conditions, particularly speed modifications. This point is important for rotating machines with discrete geometries, as in the case of pump impellers, turbo engines, and power transmissions using gears or timing belts. Traditionally, time sampling with synchronization is widely used in the measurements of various signals, which leads to a modification of frequency resolution or location when speed varies. This makes it difficult to compare the magnitude for a given frequency between two conditions.

This paper presents results from a specific development of a transmission error measurement technique in the field of geared power transmissions using optical encoders, based on the pulse timing method. Transmission Error can be defined as the angular difference between the position that the output shaft of a gear drive would have if the gearbox were perfect (without errors or deflections) and the actual position of the output shaft. Numerous works have been published on gear transmission error measurements [1-5], using either torsional accelerometers or optical

\footnotetext{
* Address for correspondence: Dr. Didier Remond, Laboratoire de Dynamique des Machines et des Structures, UMR CNRS 5006, INSA de Lyon, Bâtiment Jean d'Alembert, 18, rue des Sciences, 69621 VILLEURBANNE Cedex, France. Tel.: +33 472438941 ; Fax: +33 47243 89 30; E-mail: didier.remond@insa-lyon.fr.
} 


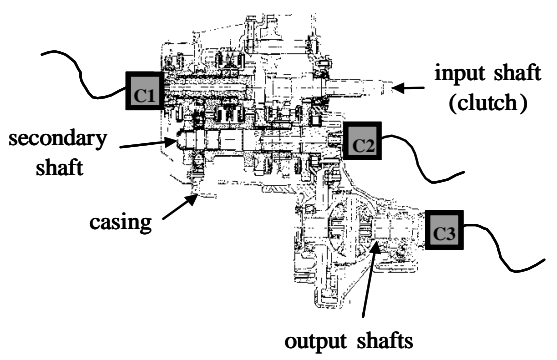

(a)

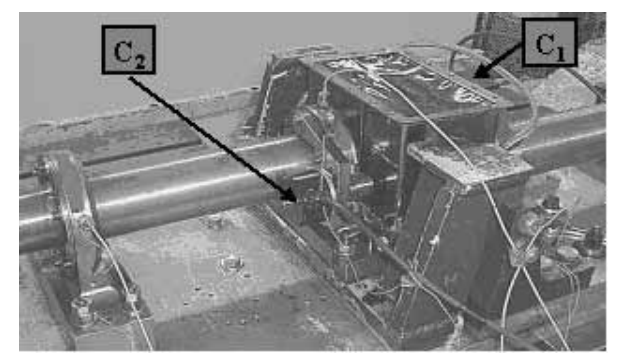

(b)

Fig. 1. Examples of application of transmission error measurements.

encoders. Under similar experimental conditions, the transmission error results obtained by these techniques are generally equally similar [6].

The use of the proposed measurement technique can be extended to a more general scope of phase difference measurement and could be applied to the case of torsional vibrations. In this approach, optical encoders are angular position sensors and can also be used as angular samplers. Consequently, in this context, three different sampling conditions of transmission error measurement using the same data set can be compared. The first set of measurements comes from an automotive gearbox (Fig. 1a), mounted on a test rig to analyze its behavior under real operating conditions [7]. Two of the three encoders mounted one on each shaft can be used to measure phase differences between corresponding shafts. The second application (Fig. 1b), concerned the fault detection on a test bench with only two shafts coupled with a single stage gear pair.

The purpose of this paper is therefore to present the benefits of angular sampling in different applications in rotating machines, all extracted from transmission error measurements, such as gearbox behavior characterization or gear fault detection and identification. First of all, angular sampling is shown to be the only manner to reach the actual magnitude of meshing frequency harmonics. Moreover, the number of pulses per revolution of encoders is not expected to be a multiple of tooth number, but only the length of the FFT window is required to be set to a proper value. Associated with classical signal processing like averaging, angular sampling is shown to be a powerful method for locating defect on discrete geometry in rotation. Finally, we present the main relevance of angular sampling which gives the possibility to compare actual meshing harmonics for different speed conditions, particularly at varying speed.

In Section 3, the pulse timing method used in transmission error measurement with optical encoders is presented. Several significant indications are recalled concerning the performances of this kind of measurement. Then, and in order to focus on the difference between angular and time sampling, the three different ways of calculating transmission error signals from data are presented.

The Section 4 recalls information and notions on angular frequency and characteristics when Fourier transformation is applied to signals obtained from angular sampling. That leads to the definition of an elementary condition for the exact localization of mesh frequency and its harmonics. Then, this condition is shown to be fundamental for obtaining a real estimation of the level of these spectral components. The difference between angular sampling and time sampling is evaluated in the case of transmission error measurement.

Afterwards, classical signal to noise improvement techniques are applied to fault detection and presented in Section 5. Here again, we emphasize that simple averaging techniques used with angular sampling on transmission error signals leads to a reliable fault diagnostic tool.

In the following section, the application of angular sampling is shown in the context of comparing different speed operating conditions. In particular, measurements are available when speed varies and a Campbell-like diagram can be obtained without distortions due to the modifications of spectral analysis characteristics induced by speed variations.

In the last section, we provide the main results and concluding remarks and we encourage experimenters to use angular sampling when measurements are required in rotating machines with discrete geometry. 


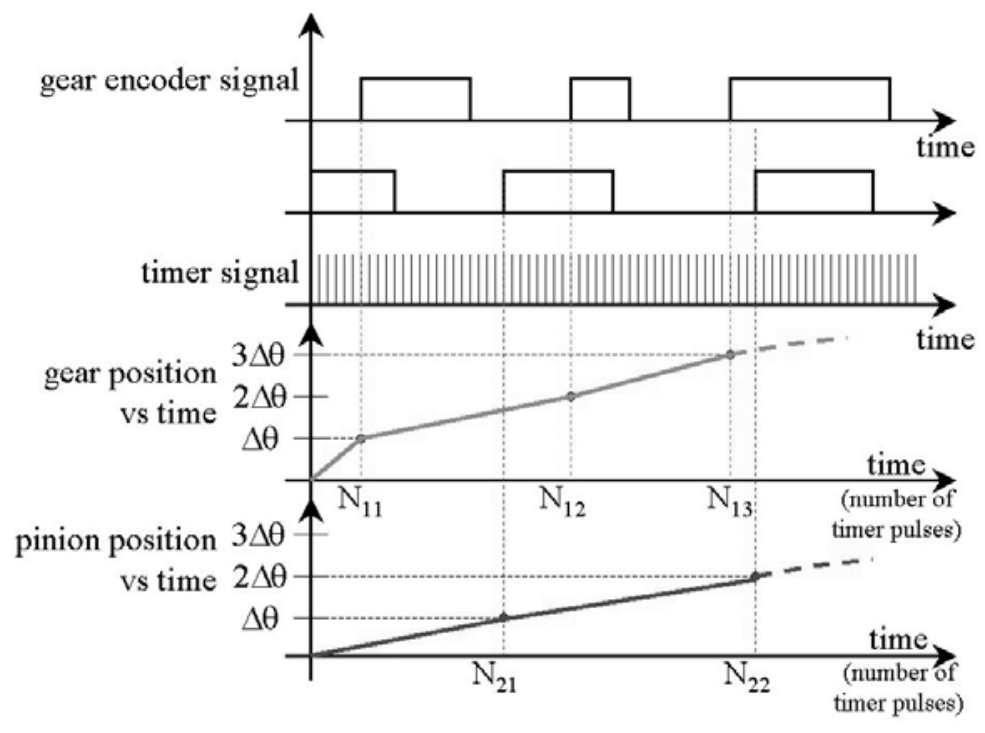

Fig. 2. Angular position of pinion (channel 1) and gear (channel 2) shafts.

\section{Transmission error measurement methods using optical encoders}

\subsection{Pulse timing method}

An elegant and well known way of reducing speed limitation problems associated with optical encoder measurements of transmission error consists in using through shaft encoders to avoid couplings and in dissociating the angular sampling and resolution from precision performance [1]. The use of through shaft encoders facilitates the integration of such measurement devices in the design of power transmissions, provided that the encoders are installed outside the power loop, in order to avoid shaft torsional effects. Through shaft encoders also allow accurate installation, and therefore minimizing misalignment and eccentricity effects.

The pulse timing method basically counts the number of pulses of a high frequency clock (timer) between the rising edges of signals generated from pinion and wheel encoders $\left(\mathrm{N}_{11}, \mathrm{~N}_{12}, \mathrm{~N}_{21}, \mathrm{~N}_{22}\right.$ in Fig. 2). It allows reliable determination of the phase difference between pinion and wheel, even when only a low number of pulses per revolution encoders are used [10].

Since the two encoders are operated in connection with the same timer and counter, the pulse timing reference can be considered as stable. The progression of the angular position of the pinion and wheel shafts can therefore be processed and simultaneous analysis of the two encoder signals allows computation of the transmission error or phase difference between the two signals. The basic principle of the pulse timing technique is presented in Fig. 2.

The theoretical precision of the pulse timing technique is determined by the fact that the minimum resolution of the difference between two events corresponds to one timer pulse. For an operating speed $\Omega$, the angular precision is given by:

$$
\Delta \theta=\begin{aligned}
& \Omega \\
& f_{h}
\end{aligned} \operatorname{rad}
$$

This relation implies that theoretical precision depends only on rotational speed, and is not affected by the resolution of the encoders (number of pulses per revolution) [1].

The number of pulses per revolution of the encoder specifies the resolution, while intrinsic encoder accuracy (i.e. grating location, jitter phenomenon or electronic signal conditioning and processing) affects measurement precision. Specific studies have been carried out to characterize this measurement precision [1,11-14], showing that it is sufficient for transmission error measurement. However, it should be remembered that encoders with a higher number of gratings will be more accurate thus, precision can be improved by using a higher number of gratings and then by dividing the generated pulse signal. Obviously, the number of pulses per revolution has to be large enough, regarding the number of gear teeth, for suitable gear harmonic analysis. 


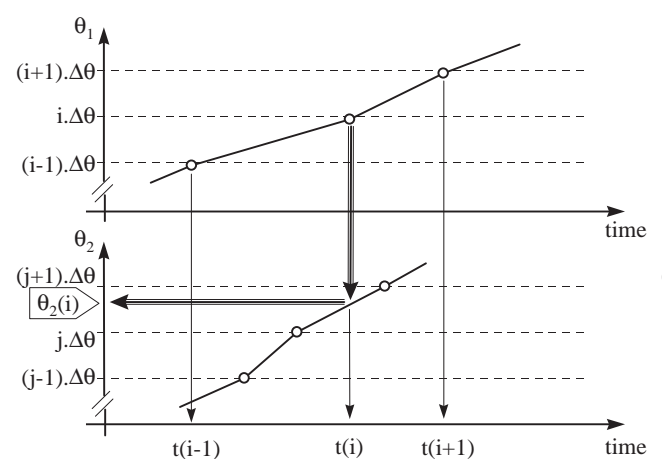

(a) Angular sampling (on pinion rising edge)

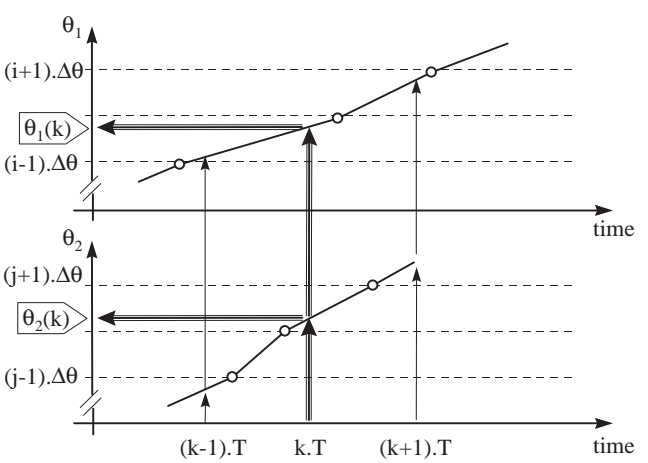

(b) Asynchronous

Fig. 3. Calculation of transmission error from encoder signals.

\subsection{Calculation of transmission error}

The basic principle of the pulse timing technique has been integrated in a dedicated data acquisition board, that permits storing the time intervals between the rising edges of both encoder signals. Therefore three different calculations can be made to estimate transmission error. The notations involved are given in Section 7.

\subsubsection{Angular sampling method}

The angular sampling method consists in performing the calculation at the rising edge either on the pinion signal (pinion rising edge) or on the wheel signal (wheel rising edge). Transmission error can be given as an angular displacement on either the pinion or wheel shaft, or as a displacement along the gear line of action. In the following, only transmission error as an angular error on the pinion shaft is used.

When an optical encoder is used on the pinion shaft, the exact time $t(i)$ of event $i$ is given by the pulse timing method and the angular position $\theta_{2}(i)$ of the wheel is solved by linear interpolation (Fig. 3a). This leads to the expression of transmission error as:

$$
\varepsilon_{a 1}(i)=\theta_{1}(i)-\frac{z_{2}}{z_{1}} \cdot \theta_{2}(i)=i \cdot \Delta \theta_{1}-{ }_{z_{1}}^{z_{2}} \cdot \theta_{2}(i)(\operatorname{rad})
$$

The transmission error signal is defined as a function of the pinion angular position and is sampled at the rate of $2 \pi$ precisely.

When an optical encoder is used on the wheel shaft, the exact time $t(j)$ of this event $j$ is given by the pulse timing method and angular position $\theta_{1}(j)$ is solved by linear interpolation. In this case, transmission error is expressed as:

$$
\varepsilon_{a 2}(j)=\theta_{1}(j)-\frac{z_{2}}{z_{1}} \cdot \theta_{2}(j)=\theta_{1}(j)-\frac{z_{2}}{z_{1}} \cdot j \cdot \Delta \theta_{2}(\operatorname{rad})
$$

where the transmission error signal is defined as a function of the wheel angular position and is sampled at the rate of ${ }_{N_{2}}^{2 \pi}$ precisely.

\subsubsection{Time sampling method}

The angular position of the two shafts at a constant sampling rate in time defined by period $\mathrm{T}$ is calculated. Time $t(k)$ is defined from the beginning of the measurement and the angular position of each shaft $\theta_{1}(k)$ and $\theta_{2}(k)$ is solved by linear interpolation (Fig. 3b). Transmission error is defined as:

$$
\varepsilon_{a s}(k)=\theta_{1}(k)-\frac{z_{2}}{z_{1}} \cdot \theta_{2}(k) \operatorname{rad}
$$

In this case, the transmission error signal is defined as a function of time and is sampled precisely at period T.

The calculation of transmission error is wholly digital while the actual gear ratio is accounted for numerically as a ratio of integers, not as an approximated real value. 

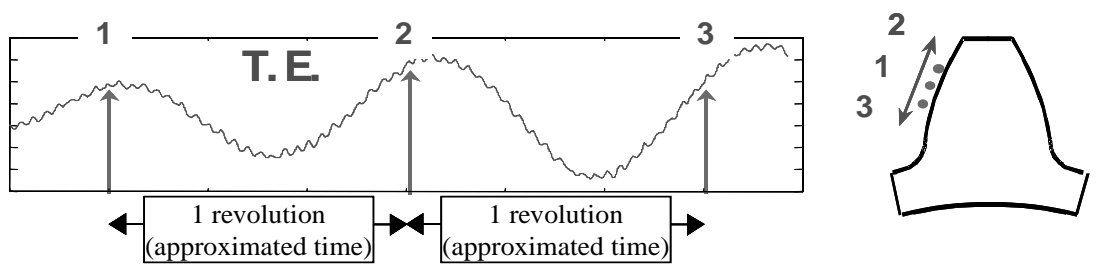

Fig. 4. Measurement points moving along the tooth profile when a signal is sampled in the time domain.

\subsection{Time sampling and speed fluctuations}

When using time sampling, the meshing points where signal is sampled are not located at the same position on teeth from one revolution to another due to the fact that transmission error induces speed fluctuations along one revolution. When eccentricity becomes substantial, these time sampled points move along the tooth profile, that leads to an intrinsic error caused by the sampling process during the measurement. In other words, this error does not allow the comparison of transmission error measurements from one revolution to another, the length of the revolution being approximated as a multiple of time-sampling periods. Figure 4 illustrates this by showing a sampling point theoretically placed at the pitch circle and that moves on the tooth flank from one revolution to another due to transmission error. Several techniques have been designed to bypass this drawback (i.e. synchronous sampling) for both measurement and signal processing. However, synchronization occurs only at the revolution period delivering a once-per-revolution signal (in the case of traditional tacho pulse), not at the time scale of tooth to tooth period. Consequently, the definition of the gear-meshing period is always approximated when time sampling is used.

On the opposite, the main advantage of angular sampling is precise and constant angular spacing between the sampling points, which are directly linked to the pinion or wheel geometry. Even if the number of pulses per revolution of optical encoders is not proportional to the tooth numbers, it remains constant throughout the revolutions during the measurement of signals. Thus, the angular sampling leads to a precise location of sampling points from one revolution to the other and provides a particular efficiency to the averaging process.

As can be seen in Fig. 5, the magnitude of eccentricity is of great importance, proving that the position of the sampling points moves when the gear tooth is engaged from one revolution to the other.

\section{Notion on angular frequency and exact mesh frequency level estimation}

The angular sampling method leads to the notion on angular wave-length, that is strongly interrelated to gear meshing. Obviously, gear meshing is an angular phenomenon caused by the periodic location of discrete meshing obstacles during revolution. Therefore the classical notion of wave-length is defined as a frequency when signals are expressed in the space domain (Fourier Transform visualization) rather than in the time domain. Figure 6 illustrates general instances of angular sampling associated with the Fourier transform in the case of angular sampling on a pinion with $z_{1}$ teeth.

All the representative periods are shown in Fig. 6 and particularly we can express the sampling length $\Delta \theta_{1}$, the length of one revolution as $N_{1} \cdot \Delta \theta_{1}$ and the length of the FFT window as $N . \Delta \theta_{1}$. These periodic characteristics correspond to frequency components, for example the sampling frequency $f_{s}^{\theta}$ or the meshing frequency $f_{1}$, expressed as:

$$
f_{s}^{\theta}=\begin{gathered}
1 \\
\Delta \theta_{1}
\end{gathered} \text { and } f_{1}=\begin{gathered}
z_{1} \\
N_{1} \cdot \Delta \theta_{1}
\end{gathered}
$$

As the frequency resolution is given by $\Delta f^{\theta}=\underset{N . \Delta \theta_{1}}{1}$, the location of gear meshing frequency or wave-length can be expressed as:

$$
f_{1}=\begin{gathered}
z_{1} \\
N_{1} \cdot \Delta \theta_{1}
\end{gathered}=z_{1} \cdot \begin{gathered}
N \\
N_{1}
\end{gathered} \cdot \Delta f^{\theta}\left(\mathrm{rad}^{-1}\right)
$$




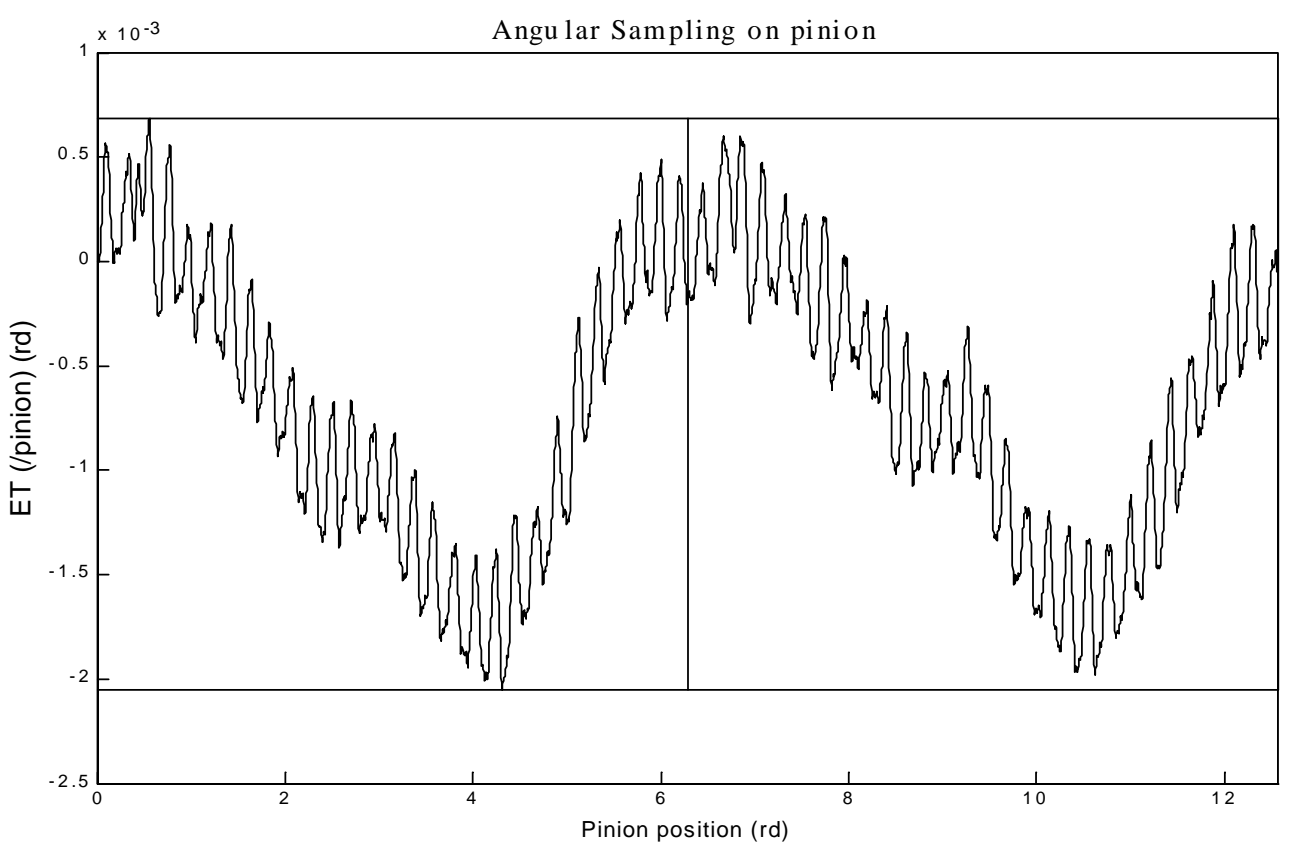

Fig. 5. Example of Transmission Error measurement (100 rpm, 90 Nm).

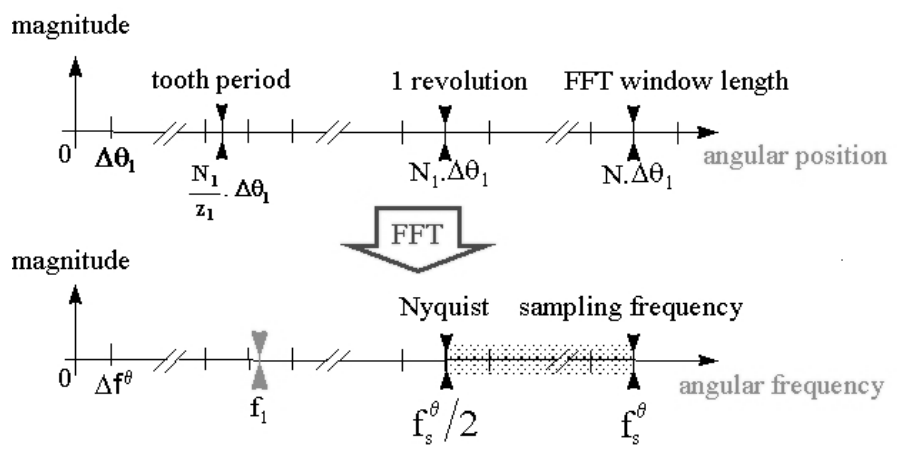

Fig. 6. Schematic representation of angular frequency or wave-length.

From Eq. (6), only one condition on the length of the FFT window $N$ needs to be set in order to obtain the mesh frequency located at an integral multiple of $\Delta f^{\theta}$, that is to say $N . z_{1} / N_{1}=k$. and Eq. (6) becomes:

$$
f_{1}=k \cdot \Delta f^{\theta} \quad\left(\mathrm{rad}^{-1}\right)
$$

This relation, which is true for any harmonic frequency of a discrete geometry, clearly implies that the number of pulses per revolution of the optical encoder does not need to be a multiple of the number of teeth to obtain an exact estimation of the meshing frequency. Once again, time sampling associated with FFT introduces errors due to both speed fluctuations and signal acquisition (including picket fence effect), which leads to the approximation of the sampled Fourier transform after signal processing. Theoretically, with time sampling, the exact location of the meshing frequency cannot be reached even if the length of the Fourier transform window becomes very large and multiple of gear period.

Several measurements were performed in order to illustrate this point with a gear pair with a 36 teeth pinion and a 38 teeth wheel, with both encoders giving $N_{1}=N_{2}=2500$ pulses per revolution. The spectral analysis, processed using the same data with different FFT window lengths is presented in Fig. $7(N=2048$ and $N=2500)$, using the angular sampling on the pinion rising edge and shows large difference in harmonics magnitude evaluation. Table 1 
Table 1

Values of $\mathrm{k}$ (equation 6) for five conditions of FFT window length and for the three first harmonics of meshing frequency

$\begin{array}{lccc} & \text { 1st harmonic } & \text { 2nd harmonic } & \text { 3rd harmonic } \\ N=2048 & k=29,4912 & k=58,9824 & k=88,4736 \\ N=4096 & k=58,982 & k=117,9648 & k=176,9472 \\ N=2500 & k=36 & k=72 & k=108 \\ N=5000 & k=72 & k=144 & k=216 \\ N=10000 & k=144 & k=288 & k=432\end{array}$

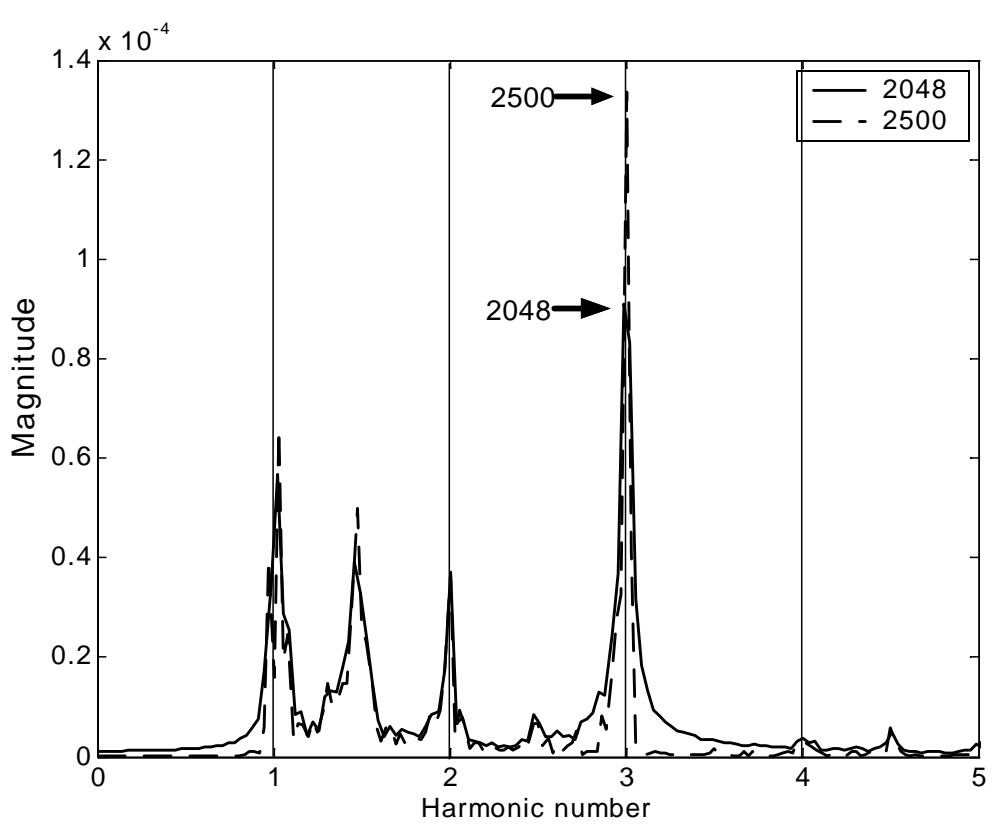

Fig. 7. Spectral analysis for two conditions in FFT window length.

gathers the associated values of $\mathrm{k}$ for the first three harmonics of the meshing frequency. Under three different lengths of FFT window, only the second harmonic is given with the same magnitude (Fig. 8a), because its location is very close to an integral multiple of the frequency resolution $(k \approx 59$ for $N=2048, k=72$ for $N=2500$ and $k \approx 118$ for $N=4096$ ). For the first and third ones, the actual level is given only when the FFT length is $N=2500$ $(k=36$ and $k=108)$. For $N=2048$, these harmonics are located respectively at $29.49 \Delta f^{\theta}$ and at $88.47 \Delta f^{\theta}$. If the length of the FFT window is increased to 4096, the third harmonic is now located at $176.947 \Delta f^{\theta}$. This point is clearly shown in Fig. 8(c) where the comparison can also be made when the length of the FFT window is equal to 5000 or 10000 points.

In order to focus on this difference and give the minimal length for a correct estimation of side-bands, three conditions were tested and presented in Fig. 8(c). As it can be seen clearly, the spectral components of interest, particularly meshing frequency harmonics and side-bands, are very well estimated with FFT window length being equal to the number of pulses per revolution of the optical encoder. In that case, the meshing harmonics are located to multiple of tooth number of the gear associated with the optical encoder which gives the sampling signal.

Progression of the meshing frequency magnitude for different values of N (FFT window length) when using either the angular sampling or the time sampling methods is compared in Fig. 9. Tests were carried out with the same raw data obtained from a gear set with $z_{1}=23$ and with a classical FFT algorithm available for any window length. The maxima of the solid curve are reached when ratio $k=N . z_{1} / N_{1}$ is an integer $(N=2000)$. For $N=2087$ or $N=2174, k$ gets close to 24 and 25 respectively after which the true mesh frequency magnitude cannot be obtained; however, a local maximum is observed. With the time sampling method, the frequency resolution problem is also shown with lower magnitudes, since time sampling devalues the periodic components of transmission error signals. 


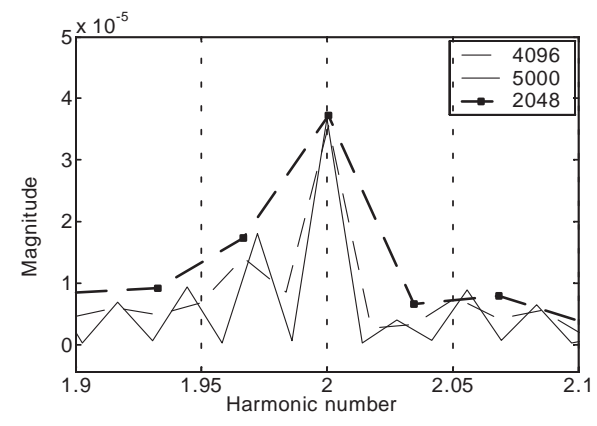

(a)

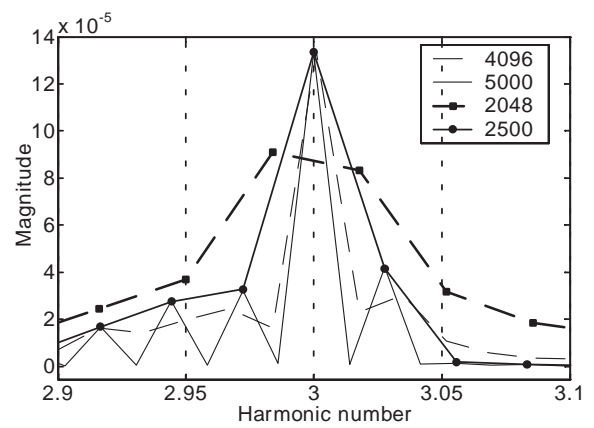

(b)

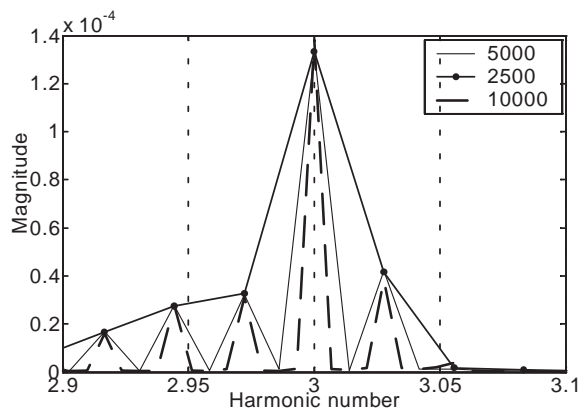

(c)

Fig. 8. Spectral analysis for different conditions in FFT window length at different harmonics.

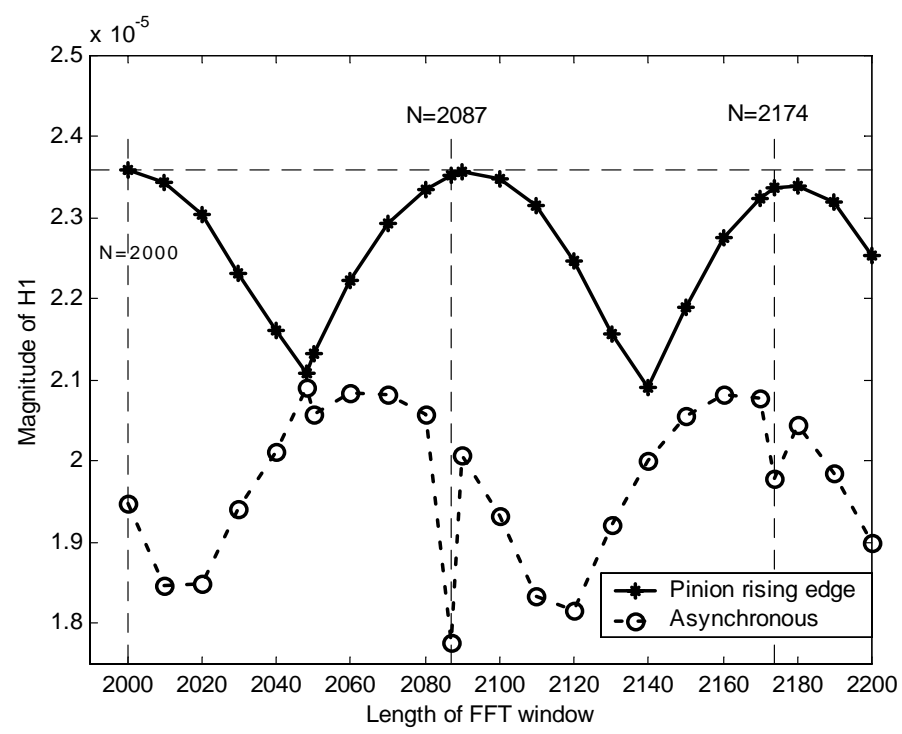

Fig. 9. Progression of mesh frequency magnitude versus length of FFT window.

The difference due to the length of the FFT window can reach $11 \%$ of the meshing frequency level when angular sampling is used and $22 \%$ when transmission error is processed using time sampling. This rough estimation can be very negative in applications such as fault detection or control in rotating machinery.

Thus, all the physical phenomena of gear meshing have to be observed from an angular description of the transmission error and from a description in terms of angular wave-length when spectral analysis is performed on the related signals. 


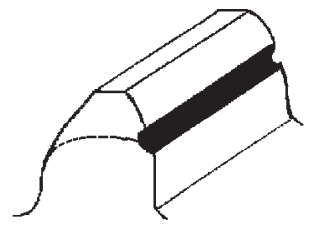

$$
\begin{gathered}
\text { width }=17.5 \mathrm{~mm} \\
\text { depth }=0.23 \mathrm{~mm} \\
\text { thickness }=0.97 \mathrm{~mm}
\end{gathered}
$$

Fig. 10. Characteristics of tested gear fault.

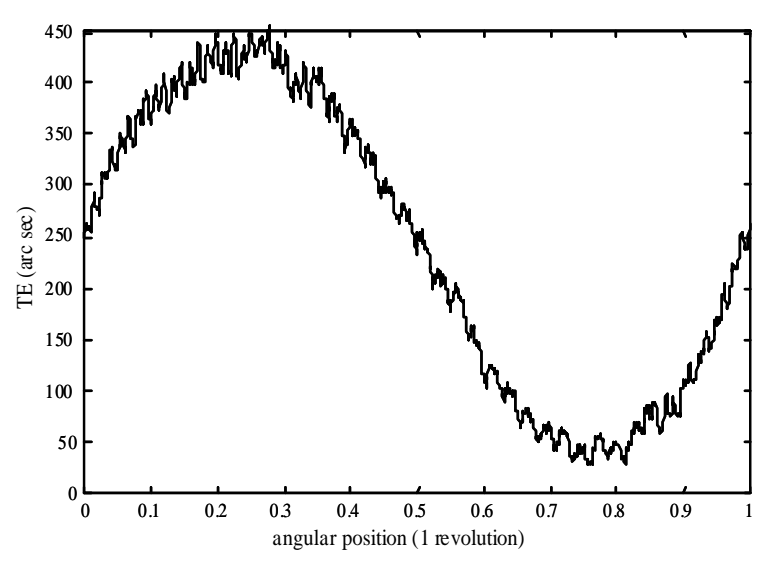

(a)

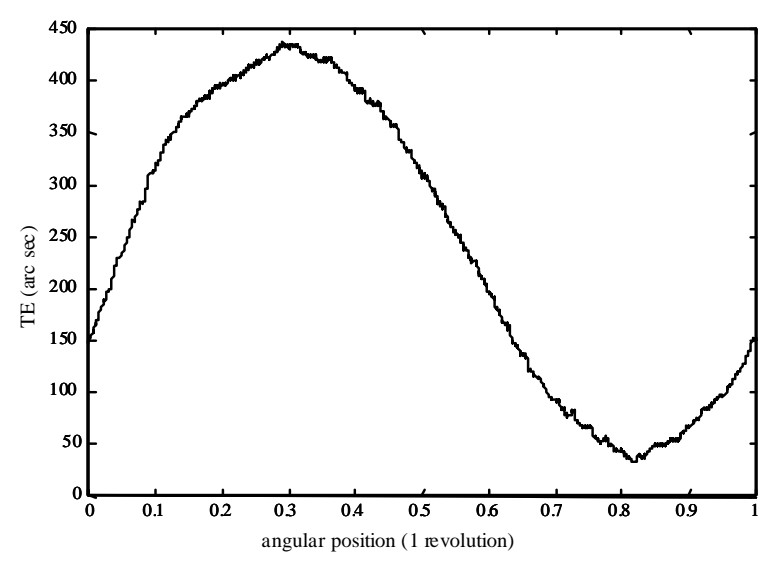

(b)

Fig. 11. Comparison between averaged pinion rising edge (a) and averaged time sampling (b) methods, on gear set with defect.

The effects of revolution speed and its fluctuations are therefore avoided and, consequently, the comparison of two transmission error signals from two speed conditions is made possible without any frequency shift or difference in the frequency location of the meshing harmonics. It is also more convenient to design filters with a cutting frequency expressed as an angular wave-length corresponding to the number of teeth on each gear set member, and then determined once and for all whatever the speed conditions.

\section{Combining angular sampling and averaging in transmission error signal analysis}

One of the classical techniques used to improve the signal to noise ratio consists in averaging a signal on a window length corresponding to the most interesting period. This process maintains the level of the deterministic and periodic components and reduces the level of the random components. Applied to transmission error signals, sampled precisely along the angular position of one of the gear set members, this technique should emphasize the behavior of each member of the gear pair studied.

In order to illustrate this simple signal process, several gear sets, fully described in [15], with identical gear geometry definition but with different levels of known defect have been tested. The results presented hereafter are from one gear without defect and another with a fault on the pitch circle, illustrated in Fig. 10.

Tests were performed on one measurement and the results are shown in Fig. 11. When the time sampling method is considered (Fig. 11b), the geometric effects of both the pinion and the wheel are not located and ordered in the time domain precisely. When performing averaging, the geometric effects are mixed together and only a low frequency component is visible after averaging at the mean period corresponding to the rotation speed of the shaft under consideration. Note that averaging is always performed with the same set of windows, starting regularly at the same point, given by the optical encoder, from one revolution to the other. Obviously, the window length is defined by the rotation speed of the shaft under consideration to cover one revolution.

On the other hand, the geometric effects of the wheel are clearly shown when angular sampling is associated with the averaging process (Fig. 11a). 


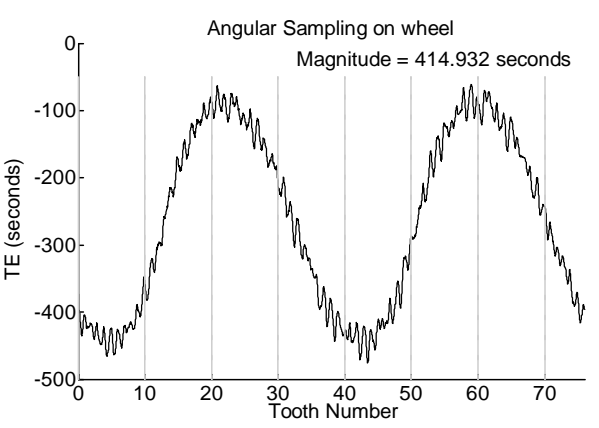

(a) averaged on wheel, no gear defect

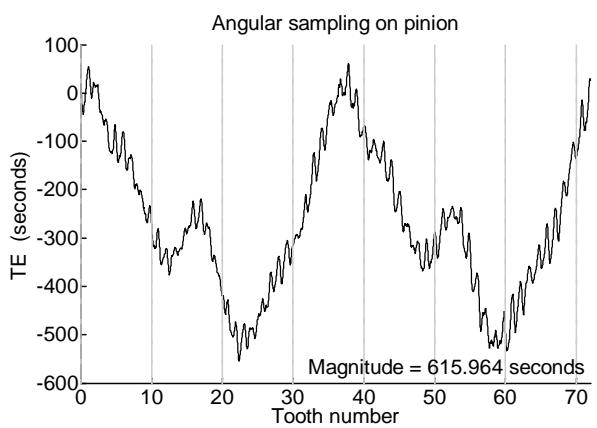

(c) averaged on pinion, no gear defect

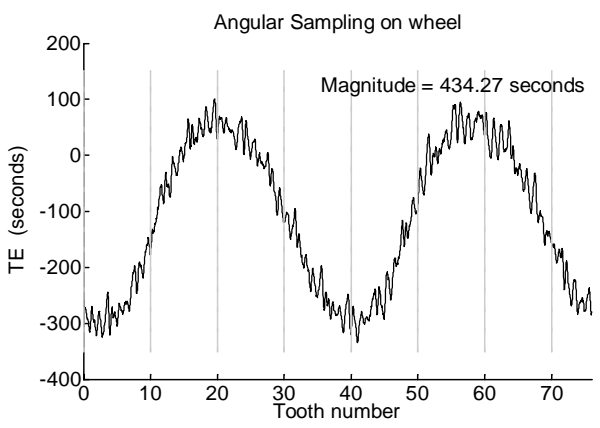

(b) averaged on wheel, with gear defect

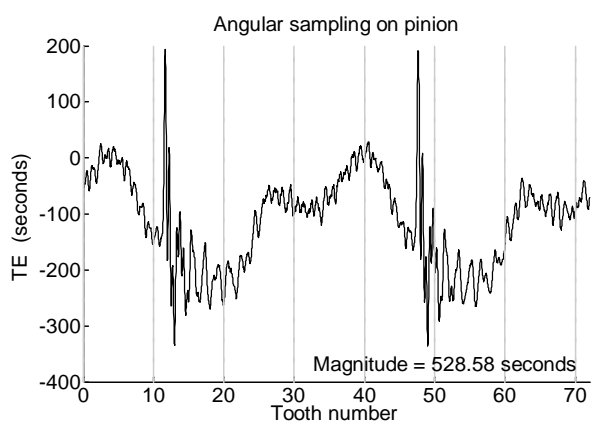

(d) averaged on pinion, with gear defect

Fig. 12. Averaged full-bandwidth signals for exactly two pinion revolutions.

By performing an average of a window length of exactly one pinion rotation (defined by the number of pulses per rotation of the corresponding encoder) on the signal sampled along the pinion position, all the geometric effects of the wheel should be spread along the averaged signal. This was done in a set of experiments to qualify gear fault detection.

Results corresponding to the two different sets of gear pairs, one without a fault (Fig. 12(a and c)) and one with the fault on the pitch circle (Fig. 12(b and d)) are compared. Transmission error signals are averaged on a window length corresponding to two revolutions of the wheel shaft (Fig. 12(a and b)), and corresponding to two revolutions of the pinion (Fig. 12(c and d)). The scales are voluntarily different to preserve the maximum amplitude of the signals. The important thing is to take into account the shape of the signals.

The effects of geometry on the wheel are shown in the full-bandwidth signal (Fig. 12a), eccentricity and toothto-tooth transmission error are visualized. Using the angular sampling method applied on the pinion signal, the dynamic effects of a defect located on the tooth flank are shown clearly as they introduce an angular vibration with a duration of about one-half revolution. Variations in the Fig. 12 are representative of the dynamic response of a classical system excited by a pulse, the vibrations are constantly damped and the tooth-to-tooth transmission error again becomes perceptible. When there is no defect on the gear tooth, the averaged signal represents the transmission error of the selected gear set member.

It is also shown that the defect on the pinion tooth introduced a disturbance in the transmission error signal on the opposite shaft. This is explained by the fact that signal averaging eliminates the tooth-to-tooth transmission error due to a difference of phase in rotation on this shaft, thus changing signal likeness from one rotation to another.

\section{Measurement of transmission error at variable speed}

Since the angular sampling method associated with FFT analysis permits a harmonic description completely independent of speed, transmission error progression can be obtained when speed varies. A schematic representation is shown in Fig. 13 when either time sampling or angular sampling are performed. In the case of time sampling 


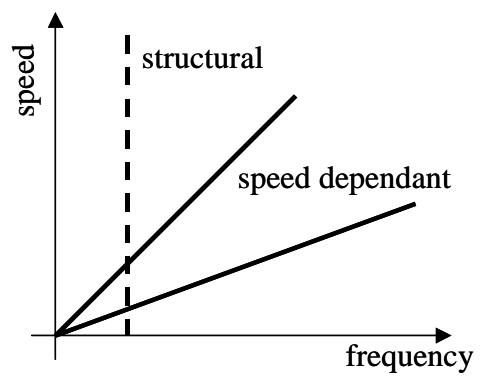

(a)

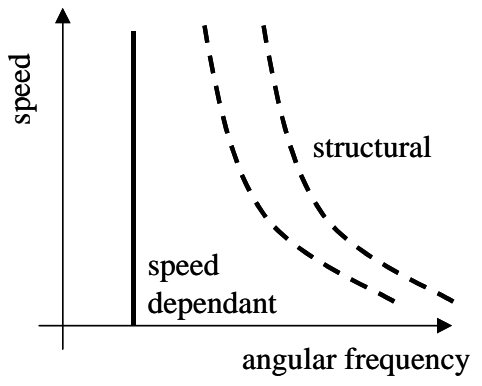

(b)

Fig. 13. Schematic representation of structural and speed dependent components.

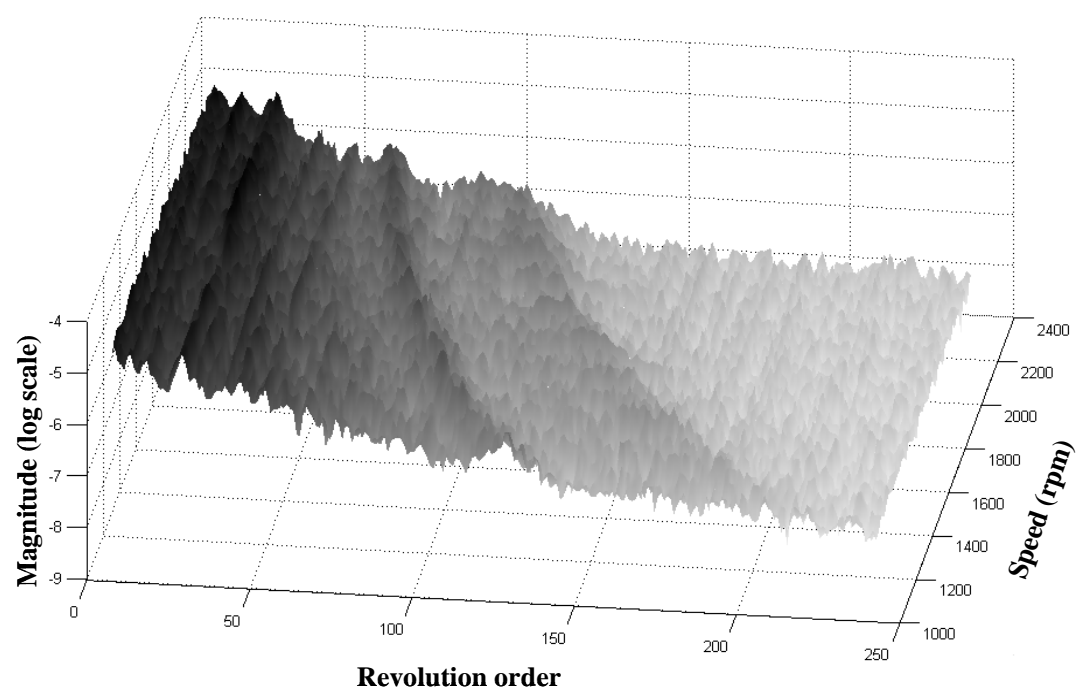

Fig. 14. Progression of transmission error with change in speed of rotation.

(Fig. 13a), Campbell diagrams provide information about frequency components: structural resonances are located as lines parallel to the speed axis. Speed dependent components appear as oblique lines in this diagram. On the other hand, in the case of angular sampling (Fig. 13b), frequency components that are speed dependent, such as meshing frequency in a geared system, are located as lines parallel to the speed axis.

Figure 14 presents a Campbell-like diagram during a decreasing speed time, with a description of angular frequency. The speed of rotation is stepped from $2000 \mathrm{rpm}$ to $1000 \mathrm{rpm}$ for about 20 seconds. The transmission error signal is divided into equally spaced slices on which an FFT analysis is performed. The mean speed of rotation is calculated from the optical encoder signals during each slice for which the FFT is performed, giving the 3D representation in Fig. 14. The angular frequency axis is graduated into a multiple of the harmonics of the pinion revolution. Thus mesh frequency and its harmonics are defined by lines parallel to the speed axis, as shown in Fig. 14.

Several effects independent from shaft speed, such as test rig resonance, appear as moving contributions along the axis of the angular harmonic number when speed is changed (Fig. 14). It is thus possible to establish the influence of increasing or decreasing shaft speed on the transmission error in order to characterize the non-linear behavior of the meshing components. 


\section{Conclusion}

The angular sampling of signals and their associated angular frequency (or wave-length) is shown to be the most suitable approach in the analysis of gear dynamics, particularly when using transmission error measurements. Signal processing and measurement apparatus based on time sampling are not suitable for transmission error measurement due to the speed fluctuations inherent to transmission error, as it modifies the contact location of sampling points along the tooth profile.

When angular sampling is used, it is possible to extract the meshing characteristics of the pinion or wheel member because the sampling points are linked to a specific location on the tooth flank and to tooth topography. Thus angular sampling is the best approach for describing the discrete geometry of meshing gears.

By satisfying a non-restricting constraint on the length of the analysis window, angular sampling permits the estimation of the true level of meshing harmonics through classical Fourier analysis. This description is independent of speed changes and associated meshing frequency shifts. It leads to the classical notion of angular frequency (or wave-length), directly associated with the discrete geometry of the gears.

Since the location of sampling points along the tooth profile is maintained in the angular sampling process, signal averaging is very powerful and magnifies events located at the same position in the averaging process. Applied to the field of tooth fault detection, the association of angular sampling and averaging is shown to be a powerful tool for separating faults located on the pinion from those located on the wheel member, only when the gear ratio is not an integer.

Regarding gear transmission error and angular sampling analysis, further investigations could be carried out using other signal processing tools.

Moreover, all the obtained results based on transmission error measurements for geared systems can be applied for measurements of all physical characteristics of rotating machines with discrete geometry (bearings, pump impellers, etc.). It would be of great interest to extend this study to other signals such as accelerations sampled on an encoder signal. The use of optical encoders opens up the possibility of synchronizing other acquisition devices in order to emphasize dynamic characteristics of rotating machines with discrete geometry.

\section{Nomenclature}

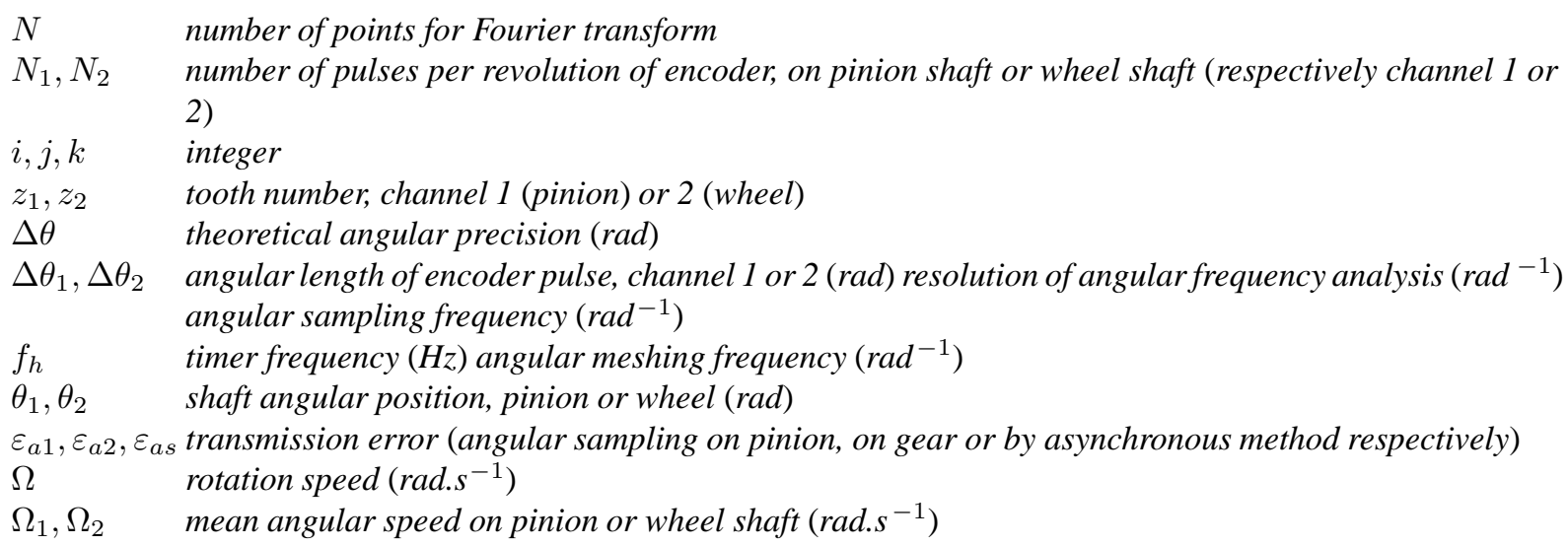

\section{References}

[1] D. Remond, Rractical Performances of High-speed Measurement of Gear Transmission error or Torsional Vibrations with Optical Encoders, Journal Measurement Science and Technology 9(3) (March 1998), 347-353.

[2] D.R. Houser and G.W. Blankenshio, Methods for Measuring Transmission error Under Load and at Operating Speeds, SAE Transactions 98(6) (1989), 1367-1374. 
[3] D. Remond and D. Play, Advantages and Perspectives of Gear Transmission error Measurement With Optical Encoders, Proc. 4th World Congress on Gearing and Power Transmissions, Paris - France, 16-18 Mar. 1999, pp. 1789-1802.

[4] J.D. Smith, A Modular System for Transmission error Measurement, Proc. of the Institution of Mechanical Engineers 202(C6) (1988), 439-442.

[5] J.D. Smith, Comparing Encoder and Accelerometer Measurement of Transmission error or Torsional Vibration, Proc. of the Institution of Mechanical Engineers, first Int. Conf. gearbox noise and vibration, 9-11 April 1990, Univ. of Cambridge.

[6] R.G. Munro, A Review of the Theory and Measurement of Gear Transmission error, Proc. of the Institution of Mechanical Engineers, 1ST Int. Conf. ON Gearbox Noise and Vibration, 9-11 April 1990, Unv. of Cambridge.

[7] D. Remond, P. Soleilhac, D. Play, P. Coppens and F. Villars, Mesures de l'Erreur de Transmission sur une boîte de Vitesses Automobile, Mécanique Industrielle et Matériaux 51(2) (Juin 1998), 61-63.

[8] C. Gosselin, T. Guertin and D. Remond, Computation and Measurement of the Kinematical Motion Error of Actual Hypoid Gears under Load, Proc. 4th World Congress on Gearing and Power Transmission, Paris - France, 16-18 Mar. 1999, $1935-1948$.

[9] C. Gosselin, T. Guertin, D. Remond and Y. Jean, Simulation And Experimental Measurement Of The Transmission error Of Real Hypoid Gears Under Load, Journal of Mechanical Design 122(1) (March 2000), 109-122.

[10] P.J. Sweeney and R.B. Randall, Gear Transmission error Measurement Using Phase Demodulation, Proc. Instn Mech. Engrs, Part C210(3) (1996), 201-213.

[11] J.D. Smith, Gear Transmission error Accuracy With Small Rotary Encoders, Proc. Instn Mech. Engrs, Part C 201(C2) (1987), 133-135.

[12] J.D. Smith, Identification of Small Amplitude Resonances in Rotary Digital Systems, Proc. Instn Mech. Engrs 202(C1) (1988), 63-65.

[13] S. Du and R.B. Randall, Encoder Error Analysis in Gear Transmission error Measurement, Proc. Instn. Mech. Engrs 212(Part C) (1998), 277-285.

[14] J.D. Smith, Alias Errors in Precision Rotary Encoder Calibration, Proc. Instn Mech. Engrs 206(C1) (1992), 71-73.

[15] J. Mahfoudh, D. Remond, C. Bard and C. Renoux, Condition Monitoring: Experimental Study of the Transmission error for the Detection of Gear Faults, Proceeding of IFToMM Conference, Darmstadt, Sept. 7-10 1998. 

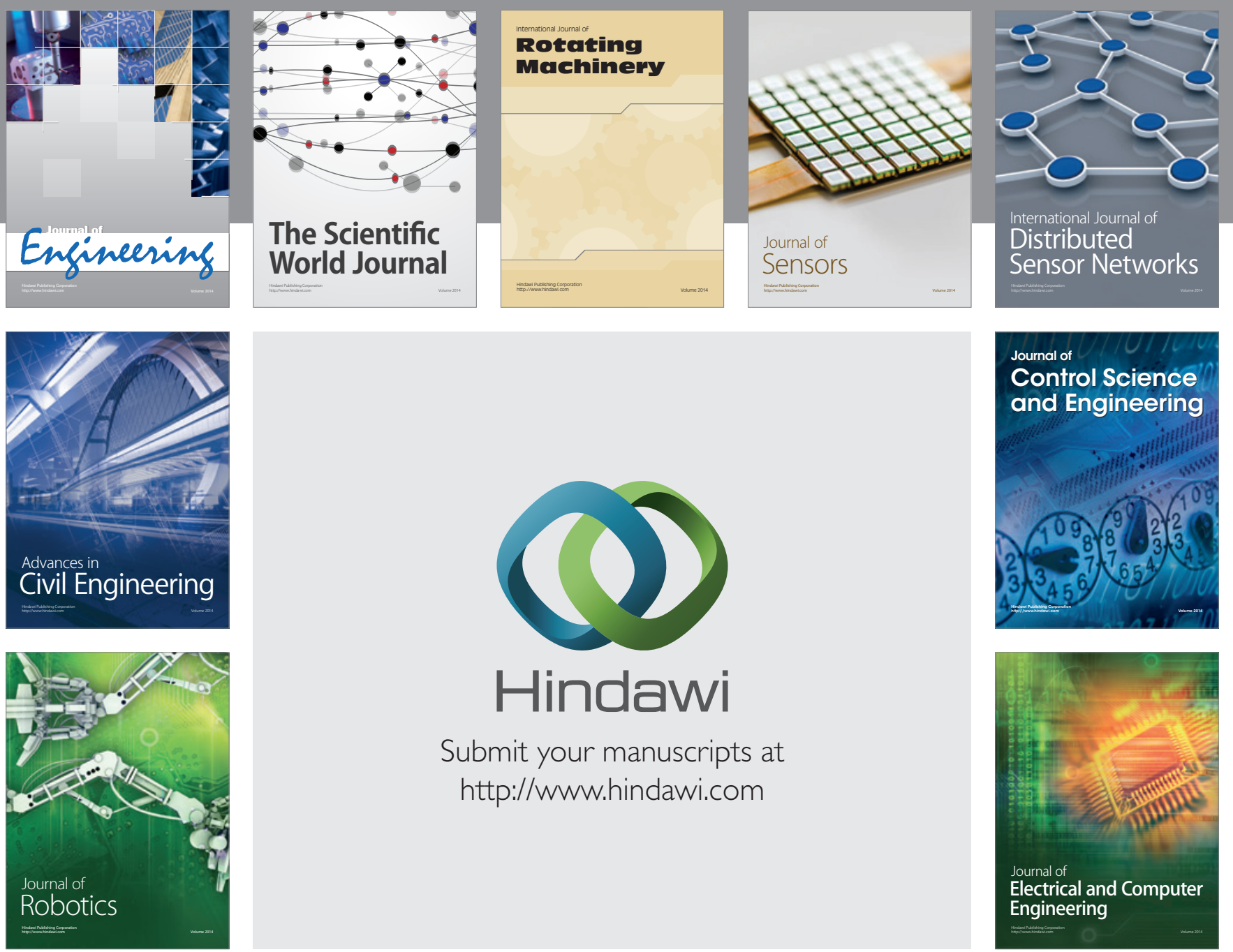

Submit your manuscripts at

http://www.hindawi.com
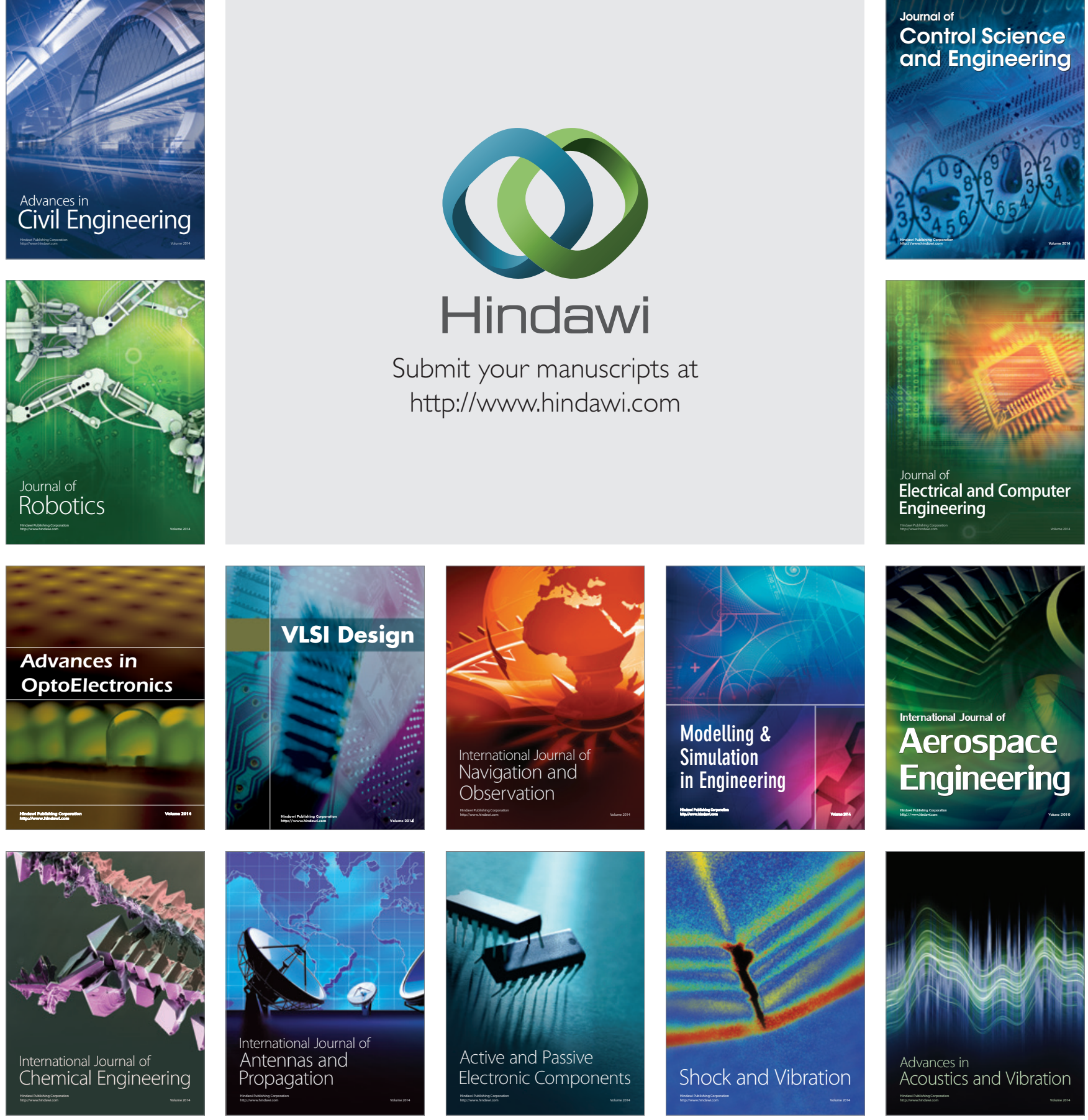